\title{
Un amigo para Frank (2012) de Jake Schreier: un robot con función sanitaria y social
}

\section{María Teresa ICART ISERN¹, Anna María PULPÓN SEGURA, Montserrat DíAZ MEMBRIVES², María Carme ICART ISERN 3}

${ }^{1}$ Facultad de Medicina y Ciencias de la Salud. Escuela de Enfermería (Universidad de Barcelona). (España). ${ }^{2}$ Hospital Duran i Reynals/HUB, Institut de Diagnòstic per la Imatge. Facultad de Medicina y Ciencias de la Salud. Escuela de Enfermería (Universidad de Barcelona). (España). ${ }^{3}$ Área Básica de Salud "Sant Josep" (Instituto Catalán de la Salud). Facultad de Medicina y Ciencias de la Salud. Escuela de Enfermería (Universidad de Barcelona). (España).

Autor para correspondencia: M Teresa Icart. Correo electrónico: $\underline{\text { mticart@ub.edu }}$

Recibido el 28 de enero de 2019; aceptado el 14 de febrero de 2019.

Cómo citar este artículo: Icart Isern MT, Pulpón Segura AM, Díaz Membrives M, Icart Isern MC. Un amigo para Frank (2012) de Jake Schreier: un robot con función sanitaria y social. Rev Med Cine [Internet] 2019;15(3): 137-145.

DOI: http://dx.doi.org/10.14201/rmc2019153137145

\section{Resumen}

El empleo de robots con finalidades diversas es una realidad que va en aumento desde los primeros prototipos industriales del siglo XIX. En este artículo se parte de la definición de robot, se aborda su evolución y algunas de sus prestaciones en los ámbitos sanitario y social. A continuación, Un amigo para Frank (2012) de Jake Schreier sirve para analizar la función que cumple un robot asistente al servicio de un anciano solitario que presenta cierto deterioro cognitivo. Finalmente, se presentan algunos ejemplos de las funciones que cumplen los robots en la atención de ancianos con diferente grado de dependencia. Estas funciones anticipan el papel de los androides como auxiliares indispensables de los cuidados del futuro tanto en el hogar como en las residencias de ancianos.

Palabras clave: robots; cine; ancianos; ciencia ficción.

\section{A friend for Frank (2012) by Jake Schreier: a robot with a healthcare and social role}

Summary

The use of robots for different purposes is a reality that is growing from the first industrial prototypes in the 19th century. This article starts with the definition of robot, discusses its evolution and some of its benefits in the health and social fields. Afterwards Frank \& Robot (2012) by Jake Schreier is used to analyze the role of an assistant robot at the service of a lonely old man who shows some cognitive impairment. Finally, some examples of the functions fulfilled by the robots in the care of elderly people with different degree of dependence are shown. These functions anticipate the role of androids as indispensable caregivers in the future both at home and in the nursing homes.

Keywords: robots; movies; elderly; science fiction. 


\section{¿Qué es y qué hace un robot?}

El término robot procede del checo "robota" que literalmente significa trabajo o labor y de modo figurado, "trabajo duro". Tradicionalmente, robota era el periodo de trabajo (seis meses al año) que un siervo debía ofrecer a su señor en la antigua Bohemia. La palabra robot fue empleada por primera vez, en 1920, por el dramaturgo Karel Capeta y dio título a su obra R.U.R. (Robots Universales Rossum) ${ }^{1}$.

Según la RAE, la palabra robot significa "máquina o ingenio electrónico programable, capaz de manipular objetos y realizar operaciones antes reservadas sólo a personas". Para Google, un robot es un "programa que explora automáticamente la red para encontrar información". La Wikipedia ${ }^{1}$ define robot como "una máquina automática programable capaz de realizar determinadas operaciones de manera autónoma y que sustituiría a los seres humanos en algunas tareas, en especial las repetitivas o peligrosas y que puede estar dotado de sensores que le permiten adaptarse a situaciones nuevas". También puede tratarse de "una persona que actúa maquinalmente y sin pensar, por inercia o por ser dirigida por otros".

Si bien los albores de los robots se remontarían al siglo IV aC cuando Arquitas de Tarento construyó un ave mecánica a la que llamó "la paloma", es con H. Tanaka (1799-1881) que se inicia el desarrollo moderno de los robots gracias a la construcción de juguetes mecánicos que servían té, disparaban flechas o trazaban los caracteres de la escritura japonesa ${ }^{1}$.

La Revolución Industrial impulsó la construcción de dispositivos automáticos diseñados para movimientos controlados y repetitivos que hasta entonces realizaban los obreros. Actualmente los robots poliarticulados (industriales) se emplean para trabajos peligrosos y/o repetitivos, en plantas de manufactura, cadenas de montaje, transporte de mercancías, limpieza de residuos tóxicos; otros se utilizan en la localización de minas terrestres, la búsqueda de personas y, más recientemente, para las exploraciones terrestres y espaciales.

La programación de un robot industrial puede ser de tipo gestual o textual. En la primera, un técnico guía al robot que va guardando los pasos a seguir hasta repetirlos de modo autónomo; mientras que en la programación textual, primero se calculan las posiciones y trayectorias que el robot debe realizar y luego se elaboran y transfieren las instrucciones a un programa que el robot ejecuta de forma autónoma ${ }^{1}$. Este tipo de robots propios de las cadenas de montaje permiten aumentar la eficiencia ya que son más precisos, rentables y resistentes que los seres humanos. Otros tienen una finalidad comercial y actúan como informadores en bibliotecas, hoteles, centros comerciales, etc.

Además de los robots poliarticulados y según su forma los robots se clasifican en: androides (forma humana), móviles (plataformas rodantes), zoomórficos y poliarticulados (brazo robótico o articulado).

El gran desarrollo de los robots zoomórficos y androides corresponde a finales del siglo XX gracias a Honda y Sony que los comercializó como mascotas y asistentes 2,3 . En medicina, Computer Motion (robot Zeus) y Intuitive Surgicals (varios modelos del robot Da Vinci), han desarrollado robots para realizar cirugía invasiva mínima, en especialidades como urología, ginecología, cirugía, ORL, etc ${ }^{4}$.

\section{¿Quién cuidará a los ancianos del futuro?}

En Europa al igual que en otros países desarrollados, la sociedad envejece a la vez que aumenta la población dependiente. Según las Naciones Unidas, si en 2017 había en el mundo 962 millones de mayores de 60 años, en 2050 serán 2.1 millones y en 2100 serán 3.1 millones los que superen los 80 años 5 .

España con una esperanza de vida media de 83,5 años (una de las más altas del mundo) y con un $20 \%$ de personas mayores de 65 años presenta una pirámide de edad con un saldo vegetativo negativo. Las previsiones del Instituto Nacional de Estadística indican que en 2033 se perderá un $10 \%$ de la población actual (se pasaría de 46,6 a 41 millones de habitantes) y la población mayor de 65 años representará el $25,2 \%{ }^{6}$.

Al margen de los efectos sobre la economía (sistema de pensiones), son obvias las consecuencias en una sociedad global cuyas preocupaciones girarán en torno a la soledad, la movilidad y la salud de una población que experimentará la "paradoja de la longevidad"7 que refiere el hecho de vivir una nueva fase de la vida que exige la incorporación de nuevas tecnologías.

Es manifiesto que para los ancianos el permanecer en su hogar el mayor tiempo posible es beneficioso a condición que sus necesidades se cubran de forma adecuada; mientras, que su ingreso en una residencia suele acarrear problemas emocionales (ansiedad, depresión, etc.) que en algunos casos se asocia a una mayor morbi-mortali$\mathrm{dad}^{8}$. Por estas razones el desarrollo de tecnologías que 
facilitan la permanencia en el hogar, entre ellas el uso de robots, puede ser un recurso de primer $\operatorname{orden}^{9}$ (Tabla 1 ).

\section{Los robots, ¿serán los futuros cuidadores?}

Los avances tecnológicos destinados a la atención de ancianos con distintos grados de dependencia podrían paliar la escasez de profesionales. Por ejemplo, en Japón se estimó que en 2015 faltaron 370.000 cuidadores para atender las necesidades especiales de sus ancianos y en Alemania se tarda 171 días en cubrir plazas de enfermeras geriátricas ${ }^{2}$.

Según K. Ishikawa, director de la residencia para ancianos Shintoni (Tokio) y una de las más innovadoras en el uso de androides, se pretende que estos reduzcan la carga laboral del personal enfermero y mejoren la eficiencia de la residencia. De este modo, la innovación robótica pretende evitar las lesiones músculo esqueléticas derivadas de la movilización de algunos pacientes. Cabe recordar que la profesión enfermera es la segunda entre las diez profesiones con mayor número de dicho tipo de lesiones ${ }^{10}$.

En 2018, el 8\% de los hogares japoneses donde residen ancianos probaron algún dispositivo robótico según un proyecto piloto impulsado por el gobierno y se prevé que en los próximos cinco años, cuatro de cada cinco ancianos recibirá algún dispositivo en su domicilio que podrá ayudarle a levantarse de la cama, recordarle una cita o la toma de medicación, lo cual le permitirá mantener una mayor autonomía11,12.

También China gana terreno en el uso de androides, desde las guarderías hasta las residencias de ancianos. En las primeras, los robots interactúan con niños de 4 a 5 años a los que les cuentan cuentos acompañados de música; en las segundas, un androide canta ópera china, llama por teléfono a los familiares y recuerda la toma de medicamentos o la necesidad de cambiar un apósito13.

En la Unión Europea, el proyecto GrowMeUp, con participación española (financiado por Horizon 2010), pretende desarrollar robots asistenciales que sirvan de ayuda y compañía a los ancianos ofreciéndoles más y mejores prestaciones que reduzcan las limitaciones físicas que pueden aislarles del entorno8,9.

\section{¿Qué pueden ofrecer los robots a los ancianos?}

En el contexto de los cuidados, cabe diferenciar las soluciones tecnológicas que ofrecen diferentes tipos de dispositivos, de las que brindan los robots. Las primeras comprenden los accesorios (destinados a corregir problemas visuales, auditivos o de movilidad), las aplicaciones informáticas disponibles en móviles, tablets, etc. (alertas de citas, medicación, aviso de caídas, etc.) y los sistemas domóticos de las "viviendas inteligentes" (detectores de humos, sensores de movimiento para detectar posibles caídas, termorreguladores y controladores del cierre y apertura de puertas y ventanas, etc.). Estas soluciones tecnológicas permiten monitorizar personas con grados leves de deterioro físico y/o cognitivo ${ }^{8,9}$.

Por su parte, los robots destinados a los ancianos pueden tener una finalidad sanitaria y/o social7,14 (tabla 1).

Los robots con finalidad sanitaria cubren necesidades de movilidad (exoesqueletos), higiene, prevención de caídas, estimulación cognitiva, manejo de medicación, control y envío de constantes vitales a profesionales. En particular, la plurimedicación que reciben los ancianos puede derivar en una dificultad de adherencia al tratamiento. A veces, de forma voluntaria los pacientes deciden abandonar o reducir las dosis por temor a la adicción (analgésicos), a los efectos secundarios o por el desconocimiento de las consecuencias de alterar las pautas; otras veces, la interrupción de la medicación se debe al olvido, a la ansiedad o a la complejidad de la pauta. La solución a estos problemas puede ser el uso de una app que recuerde la medicación; también las videoconferencias pueden ayudar en el seguimiento y control del paciente siempre que este sea capaz de gestionar los dispositivos ${ }^{15}$. En cuanto al deterioro mental de los ancianos se dispone de modelos de robots cuyo software ayuda a estimular el cerebro a través de juegos de cartas, concursos de preguntas y respuestas, materiales audiovisuales (canciones y películas que fomentan los recuerdos) ${ }^{9,16}$.

Los robots con finalidad social interactúan como una especie de asistente que puede realizar un servicio doméstico (localización de objetos, lavado de ropa, limpieza del hogar, etc.) como de compañía, lo cual mantiene la independencia y mejora la calidad de vida percibida por los ancianos ${ }^{16,17}$; es el caso de los robots zoomórficos como Paro (foca) o Aibo (perro) con los que sus propietarios combaten la soledad y la ansiedad. Algunos robots cumplen ambos cometidos, servicio y compañía, es el caso del Robot de Frank.

\section{Un amigo para un anciano: Frank \& Robot (Jake Schreier)}

El cine ha proporcionado desde Metropolis (1927) de Fritz Lang, Blade Runner (1982) de Ridley Scott, Terminator (1984) de James Cameron, RoboCop (1987) de Paul Verhoeven y sucesivos remakes, más de 200 
films en los que algún robot adquiere protagonismo. Según International Movies Data Base (IMDb), esta cifra se eleva a 5.847 títulos si la búsqueda (robot* en el título) se amplía a las series de TV y videojuegos. La tabla 2 presenta algunos de los films donde destaca la relación de los robots y la medicina.

Tabla 1. Características y prestaciones de algunos de los robots sanitarios y sociales (asistentes y de compañía) más comunes.

\begin{tabular}{|c|c|c|}
\hline País y año* & Nombre y algunas características & Prestaciones \\
\hline Estados Unidos (2004) & $\begin{array}{l}\text { PEARL. Apariencia humana con expresiones faciales. } \\
\text { Mide } 1,5 \mathrm{~m} \text {. } \\
\text { **Precio: } 100.000 \$ \text { en } 2004\end{array}$ & $\begin{array}{l}\text { Recuerda actividades diarias y citas. Monitoriza constantes vitales. } \\
\text { Sistema de telepresencia. }\end{array}$ \\
\hline Japón (2005) & $\begin{array}{l}\text { WAKAMARU. Apariencia humana con expresiones } \\
\text { faciales. Mide } 1 \mathrm{~m} \text {. } \\
\text { Precio: } 14.000 \$ \text { en } 2005 \text {. }\end{array}$ & $\begin{array}{l}\text { Gestiona agenda. Reconocimiento facial. Mantiene conversaciones senci- } \\
\text { llas. Recuerda medicación. Alerta de situaciones anómalas como caídas. }\end{array}$ \\
\hline Japón (2010) & $\begin{array}{l}\text { PARO. Foca peluda y agradable. } \\
\text { Precio: } 4.800 € .\end{array}$ & $\begin{array}{l}\text { Reduce el estrés y mejora las condiciones psicológicas. Agradece las caricias } \\
\text { ronroneando y guiña los ojos. Ayuda a calmar a pacientes con demencia. }\end{array}$ \\
\hline Japón (2010) & $\begin{array}{l}\text { DINSOW. Apariencia humana. Mide } 33,5 \mathrm{cms} \text {. } \\
\text { Precio: } 2.200 € .\end{array}$ & Asistente personal. Recuerda medicación. Responde a llamadas entrantes. \\
\hline Korea (2010) & $\begin{array}{l}\text { IRobiQ. Apariencia humana. } \\
\text { Precio: } 4.600 € .\end{array}$ & $\begin{array}{l}\text { Monitoriza constantes vitales. Recuerda medicación. Entrenamiento } \\
\text { mental. Telepresencia. }\end{array}$ \\
\hline Japón (2015) & $\begin{array}{l}\text { SOTA. Robot azul y blanco, grandes ojos negro. Mide } \\
28 \text { cms. } \\
\text { Precio: } 805 € \text {. }\end{array}$ & $\begin{array}{l}\text { Saluda. Recuerda medicación. Avisa de caídas de la cama gracias a una } \\
\text { cámara que lleva incorporada. Entabla conversaciones sencillas. Registra } \\
\text { horas de sueño y evalúa la calidad del descanso. }\end{array}$ \\
\hline Japón (2015) & $\begin{array}{l}\text { PEPPER. Robot de color blanco y ojos redondeados. } \\
\text { Aspecto simpático. Lleva una pantalla sobre el pecho. } \\
\text { Mide } 1 \mathrm{~m} \text {. } \\
\text { Precio: } 1.700 € \text {. }\end{array}$ & $\begin{array}{l}\text { Canta y mueve los brazos para que los ancianos le sigan. Capta emociones } \\
\text { a través de expresiones faciales y el tono de la voz. }\end{array}$ \\
\hline Japón (2015) & $\begin{array}{l}\text { PALRO. Pequeño perro-robot de juegos. } \\
\text { Precio: } 5.370 € .\end{array}$ & $\begin{array}{l}\text { Interactúa con usuarios a través de diferentes juegos. Cuenta con con } \\
\text { reconocimiento facial. }\end{array}$ \\
\hline Japón (2015) & $\begin{array}{l}\text { Care-O-Bot. Apariencia humana. Mide 1,5m. } \\
\text { Precio: } 2.200 € .\end{array}$ & $\begin{array}{l}\text { Ayuda a caminar. Ordena y transporta objetos. Control de hogar (tempe- } \\
\text { ratura, cierre de puertas y ventanas). Tareas domésticas (limpieza, sirve } \\
\text { comida, etc.). Recuerda medicación. Juegos de memoria. }\end{array}$ \\
\hline Japón (2016) & $\begin{array}{l}\text { RoBoHon. Apariencia humana. Mide } 19,5 \mathrm{~cm} \text { y pesa } \\
\text { 390grs. } \\
\text { Precio: } 1.610 € .\end{array}$ & $\begin{array}{l}\text { Lee y transcribe dictados. Registra caras. Sirve de teléfono móvil y como } \\
\text { proyector de imágenes y vídeos. }\end{array}$ \\
\hline China (2017) & $\begin{array}{l}\text { A-TAl. Robot simpático con antenas azules. } \\
\text { Precio: } 1.900 €\end{array}$ & $\begin{array}{l}\text { Canta opera. Llama a los familiares. Entabla conversaciones sencillas con } \\
\text { los residentes. }\end{array}$ \\
\hline Taiwán (2017) & $\begin{array}{l}\text { ZENBO. Pequeño robot doméstico con gran pantalla. } \\
\text { Costo: } 600 \text { a } 800 €\end{array}$ & Conecta a los ancianos con sus familiares. \\
\hline Japón (2018) & $\begin{array}{l}\text { AlBO. Perro mecánico. } \\
\text { Precio: } 1.700 € .\end{array}$ & \\
\hline España y Portugal (2018) & $\begin{array}{l}\text { PHAROS. Robot amigable con interfaz visual a través de } \\
\text { cámara. }\end{array}$ & $\begin{array}{l}\text { Ayuda a ancianos en actividades de la vida diaria en el hogar. Recomienda, } \\
\text { controla y evalúa el ejercicio físico que realizan ancianos. }\end{array}$ \\
\hline España (2018) & $\begin{array}{l}\text { TIAGo. Robot colaborativo y flexible. } \\
\text { Precio: } 20.000 \text { a } 60.000 € .\end{array}$ & $\begin{array}{l}\text { Localiza llaves, móvil y monitoriza el estado físico. Ayuda a acostarse, arro- } \\
\text { pa, acompaña a enfermos. Recuerda medicación. Aconseja platos saluda- } \\
\text { bles. Monitoriza constantes vitales. }\end{array}$ \\
\hline Japón (2019) & $\begin{array}{l}\text { Lovot. Apariencia de muñeco. Sensores de reconoci- } \\
\text { miento de lugares. Mide } 19,5 \mathrm{~cm} \text { y pesa } 3 \mathrm{kgs} \text {. } \\
\text { Precio: } 2.700 €\end{array}$ & Reconoce voces habituales. Sensible al tacto. Fija la mirada y parpadea. \\
\hline
\end{tabular}

*Años de su comercialización. **Los precios varían en poco tiempo, observándose su reducción con el paso del mismo. 
Tabla 2. El papel de los robots en el futuro de la medicina a través de películas de ciencia ficción.

\begin{tabular}{|c|c|c|}
\hline Título & Año, director & Aportación \\
\hline El quinto elemento/ Fifth Element & 1997, Luc Besson & Impresión en 3D de tejidos y órganos. \\
\hline Gattaca & 1997, Andrew Niccol & Modificación genética. \\
\hline Origen/Inception & 2010, Christopher Nolan & Implantación de ideas en otras personas. \\
\hline $\begin{array}{l}\text { iOlvídate de mi!l Eternal Sunshine of } \\
\text { the Spottess Mind }\end{array}$ & 2004, Michel Gondry & Alteración de la memoria, agregando o borrando recuerdos. \\
\hline $\begin{array}{l}\text { La venganza de los Sith/ Revenge of } \\
\text { the Sith (Star Wars) }\end{array}$ & 2005, George Lucas & $\begin{array}{l}\text { Robots tratan sin anestesia a un paciente con quemaduras que ha perdi- } \\
\text { do extremidades inferiores. }\end{array}$ \\
\hline Moon & 2009, Duncan Jones & Gerty con Inteligencia Artificial cuida de las heridas de Sam Bell. \\
\hline Prometeus & 2012, Ridley Scott & Robots androides que practican cirugía sobre sí mismos. \\
\hline Un amigo para Frank/ Robot and Frank & 2012, Jake Schreier & Atención de anciano con deterioro mental. \\
\hline El atlas de las nubes/Cloud Atlas & $\begin{array}{l}\text { 2012, Tom Tykwer, Lilly Wachowski, Lana } \\
\text { Wachowski, Hermanas Wachowski }\end{array}$ & Robots que diagnostican y tratan enfermedades. \\
\hline El juego de Ender/Ender's Game & 2013, Gavin Hood & $\begin{array}{l}\text { Robot cirujano diseñado por el Dr. B. Hannah Ford (universidad de } \\
\text { Washington). }\end{array}$ \\
\hline Elysium & 2013, Neill Blomkamp & $\begin{array}{l}\text { Ausencia de enfermedades gracias al radio diagnóstico que realiza che- } \\
\text { queos en segundos. }\end{array}$ \\
\hline Teorema zero/ The Zero Theorem & 2013, Terry Gilliam & $\begin{array}{l}\text { Diagnóstico de varias enfermedades con telemedicina a través del Dr. } \\
\text { Shrink-ROM. }\end{array}$ \\
\hline Dr Easy (corto) & $\begin{array}{l}\text { 2013, Jason Groves, Chris Harding, Richard } \\
\text { Kenworthy }\end{array}$ & $\begin{array}{l}\text { Robot con entrenamiento médico, negocia con un hombre armado y } \\
\text { mide las hormonas del miedo y otros parámetros. }\end{array}$ \\
\hline Lucy & 2014, Luc Besson & $\begin{array}{l}\text { Poder psico-quinético gracias a la droga } \mathrm{CPH} 4 \text { que aumenta la capacidad } \\
\text { física y mental. }\end{array}$ \\
\hline Humans (Serie TV) & 2015, Sam Vincent, Jonathan Brackley, ... & $\begin{array}{l}\text { Robots con un protocolo programado cuidan (toman TA y signos vitales } \\
\text { a través de la mirada y con el tacto les practican un escáner) de ancianos } \\
\text { que viven en su hogar. }\end{array}$ \\
\hline Big Hero 6 & 2014, Chris Williams, Don Hall & $\begin{array}{l}\text { Robots ofrecen compañía, miden parámetros y actúan como ayudantes } \\
\text { médicos. }\end{array}$ \\
\hline Interestellar & 2014, Christopher Nolan & $\begin{array}{l}\text { Tars es un robot con IA que interactúan con los humanos compartiendo } \\
\text { humor. }\end{array}$ \\
\hline
\end{tabular}

Adaptado de: Mesko B. Top 10 Science Fiction Movies about the future of Medicine. The medical futurist. [Internet]. 17 Dic 2014 [citado el 18 de enero de 2019 ]. Disponible en: https://www.youtube.com/watch?v=FmR9RIWOVjw

\section{Ficha técnica}

Título en España: Un amigo para Frank Título original: Robot and Frank (Robot \& Frank).

País: Estados Unidos.

Año: 2012.

Director: Jake Schreier.

Música: Francis Farewell Starlite.

Fotografía: Matthew J. Lloyd.

Montaje: Jacob Craycroft.

Guion: Christopher D. Ford.
Intérpretes: Frank Langella, James Marsden, Liv Tyler, Susan Sarandon, Peter Sarsgaard, Jeremy Strong, Dario Barosso, Bonnie Bentley, James D. Compton, Ana Gasteyer, Kurt Grelak, Rachael Ma, Susan Mitchell, Dana Morgan, Joshua Ormond, Jeremy Sisto, Katherine Waterston, Jesse Newman ...

Color: color.

Duración: 90 minutos.

Género: Ciencia ficción. Comedia. Drama. Comedia dramática. Robots. Amistad.

Idioma original: inglés. 
Productoras: Dog Run Pictures, Park Pictures, TBB, White Hat.

Sinopsis: "En un futuro no muy lejano, la integración de los robots en la vida humana hará posible que surjan androides programados para cuidar personas mayores en su hogar. Esa es la solución que encuentran Hunter y Madison para su anciano padre Frank, cuyas pérdidas de memoria son cada vez más frecuentes" (FilmAffinity).

Premios: Festival de Sitges: Premio del público (2012). Premios Independent Spirit: Nominada a Mejor primer guion (2013).

\section{Enlaces:}

https://www.imdb.com/title/tt1990314

https://www.filmaffinity.com/es/film983516.html

\section{Trailer}

En Un amigo para Frank, la visita de Hunter a su padre Frank, nos muestra el abandono y dejadez en que vive el anciano que, no obstante, mantiene una buena forma física. Frank se niega a ser institucionalizado y de
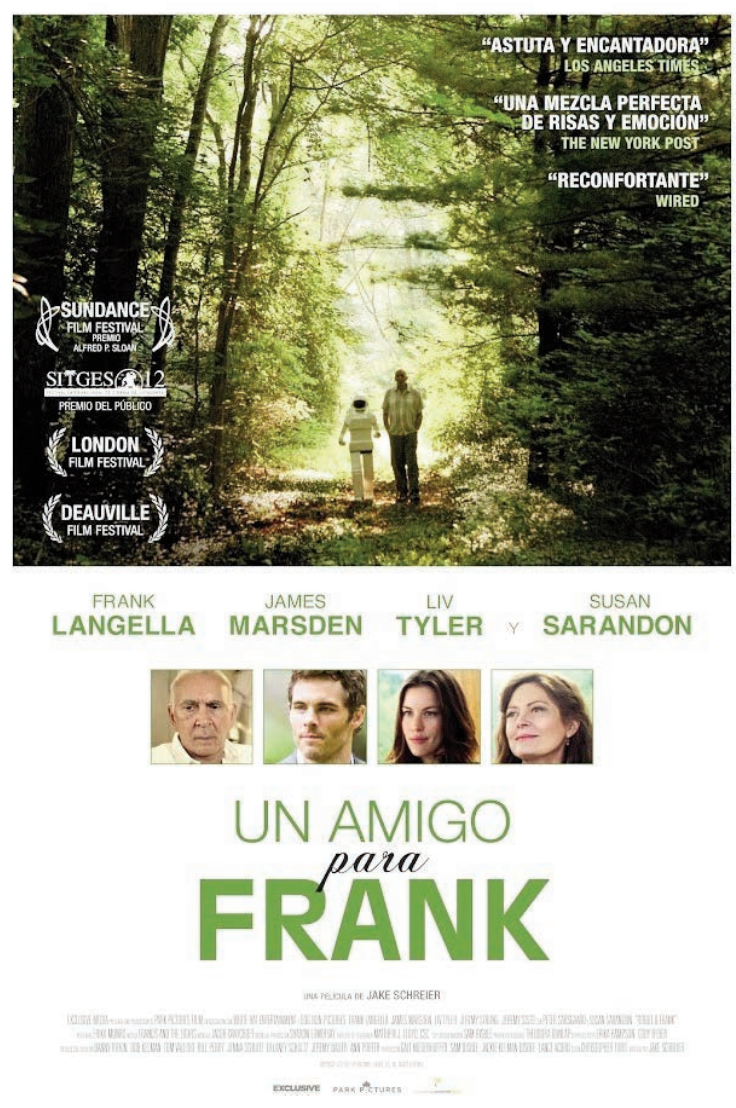

mala gana acepta el robot androide que le regala su hijo y cuyo destino es cubrir las funciones de mayordomo al servicio de Frank, que muestra signos de deterioro cognitivo (confusión, pérdida de memoria) (Foto 1).

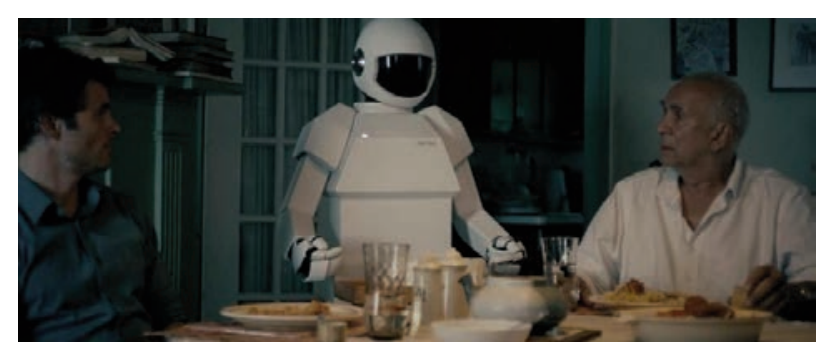

Foto 1. El Robot sirve la comida a Frank ante su hijo Hunter.

El Robot, que nunca recibirá un nombre propio, se presenta como un asistente programado para controlar y mejorar la salud física y mental de Frank. Por la mañana le despierta, le prepara un desayuno saludable, le afeita y le explica que en la vida es importante tener un proyecto, le propone cultivar un huerto, idea que el anciano rechaza (Foto 2 ).

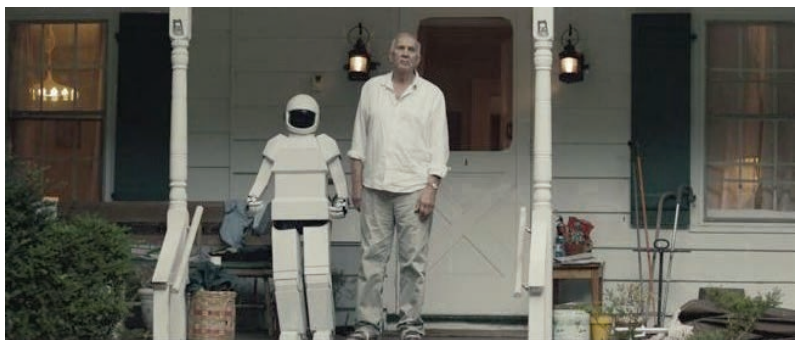

Foto 2. El Robot y Frank a la puerta de su casa.

Inicialmente, Frank desprecia los consejos y advertencias del androide al que comunica que: "prefiere morir comiendo hamburguesas que vivir a base de coliflor al vapor" (Foto 3). Pero el robot se reivindica y

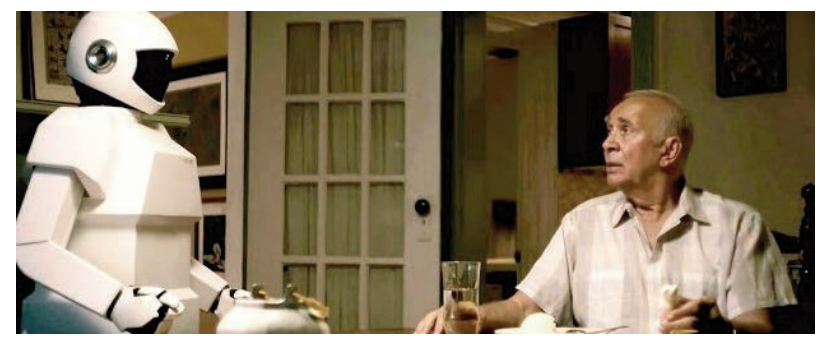

Foto 3. El Robot cumple su función asistencial preparando y sirviendo la comida. 
responde que, si Frank muere a causa de comer hamburguesas, él (el Robot) habrá fracasado y le devolverán al almacén donde borrarán su memoria.

Frank acepta que el androide le acompañe hasta la puerta de la biblioteca del pueblo donde Jennifer, la bibliotecaria, comunica a Frank el inminente cierre del lugar debido al escaso número de usuarios (Foto 4). Con paciencia y afecto le explica que los libros serán escaneados y luego reciclados; solo se salvará el volumen más valioso: una edición ilustrada de El Quijote. También Jennifer cuenta con un robot que informa sobre los libros disponibles.

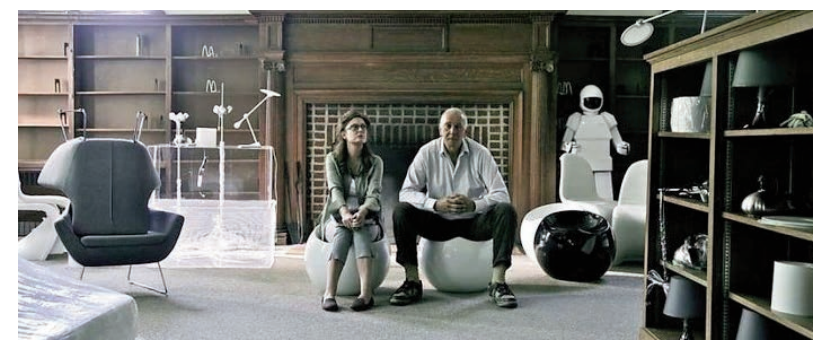

Foto 4. Frank junto a Jennifer, su esposa, en la biblioteca en plena reconversión.

El Robot continúa atendiendo a Frank quien descubre la destreza y precisión del androide en la manipulación de cerraduras, una habilidad que forma parte del pasado delictivo del anciano. Este se reivindica como un especialista en joyas de alta gama que, condenado por robo y evasión fiscal, considera que su actividad sólo ha perjudicado a los sinvergüenzas de las aseguradoras.

Cuando Frank pide al Robot que defina el término robar, este lo hace con precisión, sin embargo, manifiesta no tener opinión sobre el bien y el mal, afirma que sabe de definiciones pero no las entiende. En esa misma línea, el androide aclara que no está vivo, que no existe, que solo responde a un programa, tales afirmaciones parecen entristecer a Frank.

Frank acabará defendiendo el valor del Robot ante el rechazo de su hija, Madison (Foto 5), que desde el inicio ha sido contraria a la máquina. Por eso cuando la joven trata al androide como a una máquina a la que desconecta, Frank protesta y argumenta que "el Robot no es un criado, no se le puede encender y apagar, sin más", y acaba afirmando que necesita al Robot porque "es su amigo"18-20. Ante el progresivo deterioro mental de Frank, el Robot manifiesta su preocupación, al observarle excitado le advierte que tal vez deba sedarle y, finalmente, contacta con Hunter.

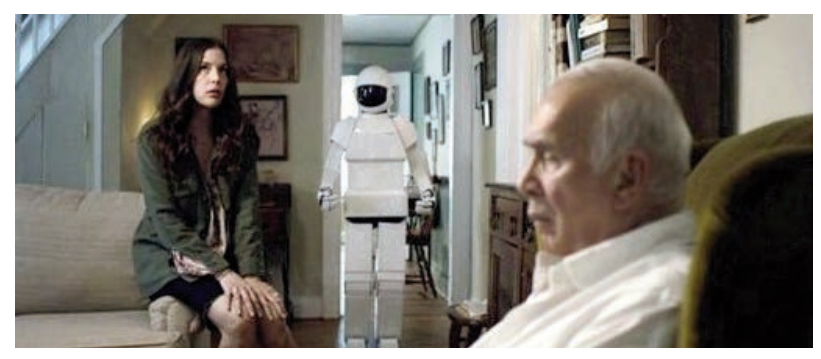

Foto 5. Frank junto a su hija ante la cual confiesa que el Robot es su amigo.

Cerca del final, en la escena en que Frank irrumpe en el despacho de Jennifer descubrimos la razón del cariño que existe entre ambos; allí reconoce antiguas fotografías de él junto a Jennifer, su esposa, y sus hijos, Hunter y Madison. En la última secuencia vemos a Frank en una residencia junto a otros ancianos acompañados de robots similares a su Robot que ha sido desconectado.

Los títulos de crédito se acompañan de imágenes de robots que realizan tareas domésticas (abren y cierran puertas, recogen objetos, manipulan tostadoras, lavavajillas y lavadoras, etc.), interaccionan con personas (juegan al ajedrez, conducen sillas de ruedas, sirven bandejas con comida y bebidas, visten y levantan pacientes) e incluso van en bicicleta de forma autónoma.

\section{¿Cómo ayuda el Robot a Frank?}

Frank no presenta problemas de movilidad ni riesgo de caídas, pero sí dificultades para realizar algunas actividades de la vida diaria (preparación de comidas, higiene, etc.) y otras de tipo instrumental (manejo de dinero, compras, orientación, etc.). El deterioro cognitivo de Frank es evidente, como lo demuestra la pérdida de memoria, la dificultad para razonar, los episodios de confusión y agitación. Al igual que muchos ancianos no quieren reconocer su deterioro ${ }^{18,19}$.

Frank utiliza gafas y se maneja bastante bien con su móvil y es capaz de comprender las posibilidades de colaboración de su Robot que realiza tareas como las de cortar el césped, servir comida, sacar el polvo, acompañarle a la biblioteca y en la vigilancia de la vivienda que pretende asaltar, etc. Además es capaz de detectar la ansiedad de Frank y de actuar de inmediato avisando a su hijo.

El control y supervisión del anciano podría venir de amigos (no es el caso del malhumorado Frank) o de familiares, como es el caso de Frank que recibe las visitas semanales de su hijo y, esporádicamente, las de su hija. También su esposa, a quien ya no reconoce, le cuida a 
cierta distancia, gracias a lo cual se va retrasando el ingreso del anciano en una residencia. Frank no recibe atención o ayuda por parte de ningún servicio socio-sanitario, algo compatible con el país donde se desarrolla el film ${ }^{20}$.

El Robot de Frank se convierte en su amigo y acaba despertando el afecto del anciano que, carente de vida social, siente afecto por el androide convertido en el compañero de su soledad.

\section{¿Qué significa aceptar un robot?}

Si bien las tecnologías ofrecen múltiples formas de comunicación (móviles, internet, WhatsApp, email, etc.) es evidente que su uso exige un mínimo de habilidades que algunos ancianos ya no son capaces de adquirir o de mantener con el paso de los años. Sin embargo, cuando son capaces de adquirir esas destrezas las emplean constantemente porque les permiten una mayor intimidad; en ocasiones, sea por vergüenza, orgullo o estoicismo los ancianos no reconocen su deterioro funcional (incontinencia, hipoacusia, pérdida de memoria, etc.) que consideran algo natural y consubstancial al envejecimiento, entonces preguntar a Google o a alguna app sobre su problema de salud puede ser menos embarazoso que hacerlo a un profesional.

Algunos robots pueden facilitar el contacto del usuario con los familiares, amigos y profesionales. Si bien se acepta que la robótica puede ayudar a la atención de personas mayores no es menos cierto que plantea un buen número de cuestiones éticas si se pretende emplear en sustitución de los cuidadores humanos ${ }^{21,22}$.

La primera barrera que debe superar el uso de robots es la de su aceptación por parte del usuario siendo fundamental que el robot sea percibido como un elemento útil, entretenido, fiable y fácil de utilizar. La aceptación también depende de su apariencia, según algunos estudios los ancianos muestran mayor predisposición a cumplir las órdenes (hacer ejercicio físico, seguir un tratamiento, etc.) de los robots con aspecto formal y serio que las procedentes de los que recuerdan juguetes infantiles. Esa podría ser la razón del fracaso de Hopis que medía la glicemia, la temperatura y la tensión arterial (enviaba estos parámetros al médico o a la enfermera) o de Ifbot que ofrecía juegos y tablas de ejercicio físico 9,16 , en ambos casos su apariencia de mascotas disgustó a los ancianos que se sentían tratados como niños. Por el contrario, otras veces los robots zoomórficos satisfacen necesidades afectivas (acariciar y mimar) y sustituyen a una mascota real a la que el anciano no podría cuidar.
En todo caso, los robots deben estar diseñados a medida y cumplir las funciones deseadas por el destinatario, entre las que destaca: detectar caídas, recordar la toma de medicación, realizar llamadas telefónicas, monitorizar constantes vitales, contactar con familiares y profesionales de la salud. Si además conecta con las necesidades emocionales, el robot androide obtiene mejores resultados que las apps disponibles en un móvil o una tablet ${ }^{23,24}$. No obstante, la aceptación de los robots es dispar: los androides son mejor aceptados por los ciudadanos japoneses que por los británicos o estadounidenses.

En resumen, a medida que se envejece es habitual que se requiera ayuda para realizar tanto las actividades instrumentales como las de la vida diaria. Las tecnologías y los robots pueden ayudar a mantener la independencia y retrasar el ingreso en centros asistidos. Las ventajas de los robots van desde que pueden ser programados para monitorizar constantes vitales, realizar tareas domésticas a la vez que ofrecer información y apoyo. El reto de los robots del futuro es que fomenten actividades que promuevan los hábitos saludables, para ello la investigación debe considerar las necesidades de los usuarios y desarrollar programas que estos puedan gestionar sin que la brecha digital sea un obstáculo.

\section{Referencias}

1. Colaboradores de Wikipedia. Robot [Internet]. Wikipedia, La enciclopedia libre [Internet]. 28 de diciembre de 2018 [citado el 18 de enero de 2019].

2. Hurst D. Japan lays groundwork for boom in robot carers. The Guardian [Internet]. 6 de febrero de 2018 [citado el 18 de enero de 2019].

3. Aguilera-Hellweg M. Los robots están aquí. Robots capaces de pensar, actuar y relacionarse con nosotros. ¿Estamos preparados? The National Geography [Internet]. 1 de Agosto de 2011 [citado el 18 de enero de 2019]; 10(1).

4. Tomlinson Z. 15 Medical Robots that are Changing the World. Interesting Engineering [Internet]. 11 de octubre de 2018 [citado el 18 de enero de 2019].

5. Bharadwaj R. Applications of Artificial Intelligence in Elderly Care Robotics. Emerj [Internet]. 10 de febrero de 2019 [citado el 18 de enero de 2019].

6. Instituto Nacional de Estadistica. Proyecciones de Población 2018. INE [Internet]. 5 de noviembre de 2018 [citado el 18 de enero de 2019].

7. Marcos Z, Ruiz del Árbol M. Robots que nos ayudarán a cuidar de nuestros mayores. El País [Internet]. 1 de marzo de 2018 [citado el 18 de enero de 2019]; 10(1): 37-44.

8. Scocco P, Rapattoni M, Fantoni G. Nursing home institutionalization: a source of eustress or distress for the elderly? Int J Geriatr Psychiatry. 2006; 21 (3):281-7.

9. Robinson H, MacDonald B, Broadbent E. The Role of Healthcare Robots for Older People at Home: A Review. Int J of Soc Robotics. 2014; 6 (4): 575-91.

10. Cheung K, Szeto G, Kin Bun Lai G, Ching SY. Prevalence of and Factors Associated with Work-Related Musculoskeletal Symptoms in Nursing Assistants Working in Nursing Homes. Int J Environ Res Public Health. 2018; 14(2): 123-33. 11. Foster M. Aging Japan: Robots may have role in future of elder care. Reuters [Internet]. 28 de marzo de 2018 [citado el 18 de enero de 2019].

12. Díez PM, Japón confía en la última generación de robots para cuidar a los ancianos. ABC Sociedad [Internet]. 1 de octubre de 2017 [citado el 18 de enero de 2019].

13. Chen S. Inside china's experiment to find friends for 230 million old people. 
Classic.qz. [Internet]. 1 de marzo de 2014 [citado el 18 de enero de 2019]; 10(1): 37-44.

14. García J. Los robots con más corazón: ya cuidan a los más mayores y a los más enfermos. La Información [Internet]. 23 de enero de 2017 [citado el 18 de enero de 2019].

15. Zamir S, Hennessy CH, Taylor AH, Jones RB. Video-calls to reduce loneliness and social isolation within care environments for older people: an implementation study using collaborative action research. BMC Geriatr. 2018; 2 (18):62.

16. Elkan R, Kendrick D, Dewey M, Hewitt M, Robinson J, Blair M, Williams D, Brummell $K$ Effectiveness of home based support for older people: systematic review and meta-analysis. BMJ 2001; 323(7315)719-25.

17. Broekens J, Heerink M, Rosendal H. Assistive social robots in elderly care: a review. Gerontechnology 2019;8(2):94-103.

18. Caviaro JL. 'Un amigo para Frank', la humanidad del robot. Espinof [Internet]. 28 de mayo de 2013 [citado el 18 de enero de 2019].

19. Cuéllar JM. Crítica de "Un amigo para Frank»: Amistad más allá de la memoria. ABC.es [Internet]. 24 de mayo de 2013 [citado el 18 de enero de 2019].

20. Torras C. Un amigo para el ser humano. El Cultural [Internet]. 12de enero de 2018 [citado el 18 de enero de 2019].

21. Sharket AJ. Granny and the robots: ethical issues in robot care for the elderly. Ethics and Information Technology. 2010;14(1):27-40.

22. Stahl BC, Coeckelbergh M. Ethics of health care robotics: Towards responsible research and Innovation. Robotics and Autonomous Systems 2016; 86:152-61.

23. Siegel C, Hochgatterer A, Dorner TE. Contributions of ambient assisted living for health and quality of life in the elderly and care services - a qualitative analysis from the experts' perspective of care service professionals. MC Geriatr. 2014.14:112.

24. McLoughlin M. Lovot, el robot creado para dar amor. El Confidencial [Internet]. 1 de marzo de 2014 [citado el 18 de enero de 2019]; 10(1): 37-44.
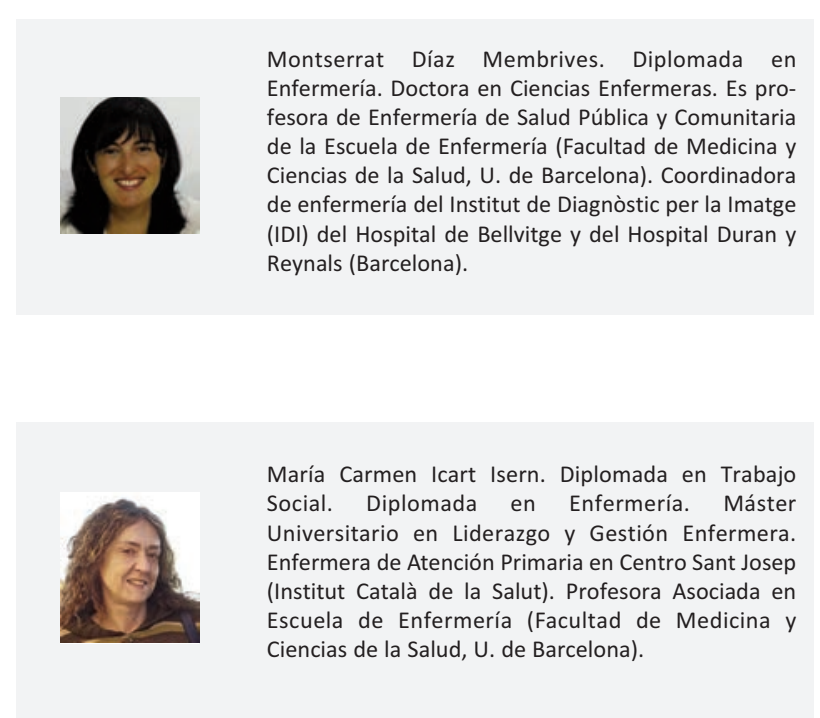

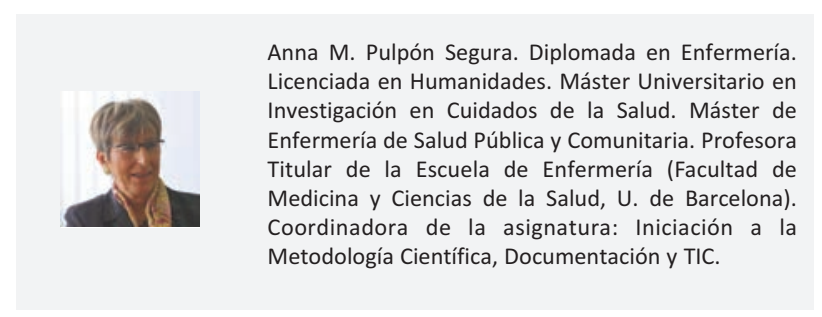

María Teresa Icart Isern. Diplomada en Enfermería. Doctora en Medicina y Cirugía. Máster en S. Pública. Catedrática en Enfermería Comunitaria y S. Pública en la Escuela de Enfermería (Facultad de Medicina y Ciencias de la Salud, U. de Barcelona). Coordina las asignaturas de Investigación Clínico-epidemiológica, Comunicación Científica y Evidencia Científica. Es coautora de: Films in Health Sciences Education. Learning through moving images (Ed:UB, 2012) y de Enfermeras en el cine (Ed: UB, 2015). 